ZOOLOGY

\section{Bee trade spreads parasites}

The international trade in beehives distributes dangerous parasites around the world.

William Hughes at the University of Sussex, $\mathrm{UK}$, and his colleagues investigated 48 commercially produced hives of bufftailed bumblebees (Bombus terrestris). Despite being certified as parasite-free by their producers, more than $75 \%$ of the hives carried microbial parasites. Uninfected bees that were fed faeces or pollen collected from the commercial hives subsequently tested positive for parasites and had shorter lifespans.

Worldwide, more than one million bumblebee hives are shipped every year, and the authors suggest that imported hives could infect native pollinators.

J. Appl. Ecol. http://dx.doi. org/10.1111/1365-

2664.12134 (2013)

\section{MATERIALS SCIENCE}

\section{Odd performance under pressure}

When squashed down, most materials expand outwards, but one class of 'metamaterials' gets narrower instead.

Katia Bertoldi at Harvard University in Cambridge, Massachusetts, and her colleagues crafted several structures using arrangements of spherical shells with regularly spaced holes. Whereas most structures are engineered to avoid mechanical instabilities, this metamaterial exploits them

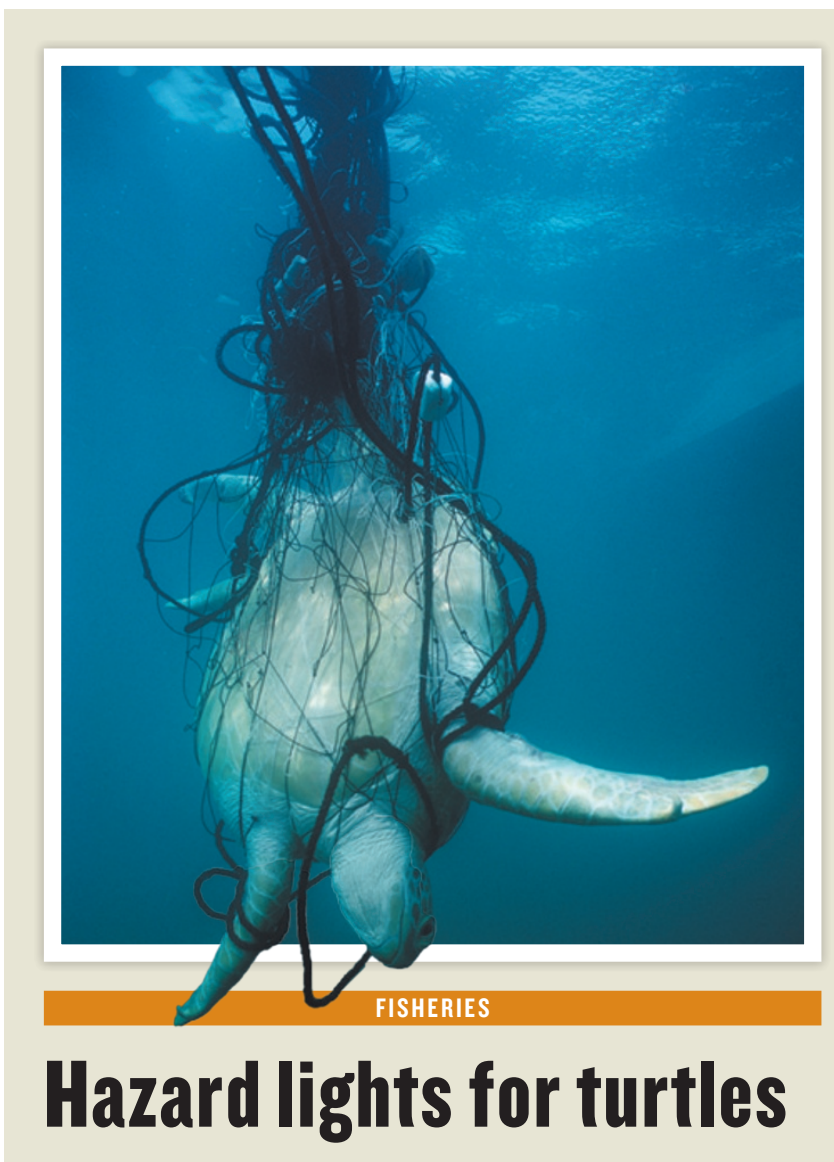

Nets that are lit by ultraviolet lights deter turtles but catch just as many fish as unlit nets.

Vulnerable sea-turtle species are often caught unintentionally in nets set out by fisheries (pictured). Many commercial fish species cannot see ultraviolet light, but several types of sea turtle can, so a team of researchers led by John Wang at the University of Hawaii in Honolulu tested whether visual cues stopped turtles from being trapped. The group placed ultraviolet lights every 5 metres along net floatlines and compared catches to those from unlit nets. The lights reduced the capture rate of green sea turtles (Chelonia mydas) by $40 \%$ and, in separate studies in a commercial fishery, did not significantly alter the value or number of marketable fish caught.

Biol. Lett. 9, 20130383 (2013) by buckling and folding to become narrower under compression before returning to its original shape after the pressure is removed. $\mathrm{X}$-ray images and numerical simulations showed how the holes in the structures (one example pictured) distort under increasing strain.

These reversible architectures could be useful in applications such as energy-absorbing materials or acoustic dampeners, the authors suggest.

Adv. Mater. http:// dx.doi.org/10.1002/ adma.201301986 (2013)
REMOTE SENSING

\section{Counting carbon} from above

An airborne method for measuring biomass in forests and other ecosystems is as accurate as ground-based studies and can be used to create a national carbon map.

Gregory Asner at the Carnegie Institution for Science in Stanford, California, and his colleagues measured vegetation across $2.4 \%$ of Panama using an aeroplane equipped with a laser-based surface-detection system. They calibrated results by comparing data with 228 field plots and scaled up their analysis using satellite observations.

This produced the first high-fidelity map of carbon stocks across an entire country. The map has an uncertainty of $10 \%$ in areas measured from the plane, and $20 \%$ on average in other areas. The authors suggest that laser-based data can fill in gaps where there are no field data.

Carbon Balance Mgmt. 8, 7 (2013)

IMMUNOLOGY

\section{Antibody tutors travel}

A group of specialized white blood cells migrates back and forth through lymph nodes to teach other immune cells how to make a greater variety of antibodies against invading bacteria and viruses.

A team led by Michel Nussenzweig at the Rockefeller University in New York City and Gabriel Victora at the Whitehead Institute for Biomedical Research in Cambridge, Massachusetts, used fluorescence microscopy to monitor the behaviour of specialized T cells in lymph 